\title{
Molecular Cloning, Characterization, and Expression Analysis of DROOPING LEAF gene in the Pistillody Line of Common Wheat
}

\author{
Li Zhang ${ }^{1}$, Zaijun Yang ${ }^{1}$, Zhengsong Peng ${ }^{1}$, Yan Yu ${ }^{1} \&$ Qingxu Sun ${ }^{1}$ \\ ${ }^{1}$ The Key Laboratory of Southwest China Wildlife Resources Conservation, College of Life Science, China \\ West Normal University, Nanchong, Sichuan, China \\ Correspondence: Zaijun Yang, The Key Laboratory of Southwest China Wildlife Resources Conservation, \\ College of Life Science, China West Normal University, Nanchong, Sichuan, China. Tel: 86-172-568-352. \\ E-mail: zaijunyang@cwnu.edu.cn
}

Received: May 4, 2015

Accepted: September 3, 2015

Online Published: November 30, 2015

doi:10.5539/mas.v9n13p44

URL: http://dx.doi.org/10.5539/mas.v9n13p44

\begin{abstract}
In this article, we report the cloning, characterization, and expression pattern of Triticum aestivum DROOPING $L E A F$ gene $(T a D L)$ in pistillody line of common wheat. Three $T a D L$ homoeologous genes $(T a D L 1, T a D L 2$, and $T a D L 3$ ) were isolated and found to be located on chromosomes 4AS, 4DL, and 4BL, respectively. The putative $T a D L 1, T a D L 2$, and $T a D L 3$ protein were $22.4,22.6$, and $22.3 \mathrm{kDa}$, with theoretical $\mathrm{pI}$ of 9.04, 9.04, and 8.98 and containing two distinct domains, namely, a zinc-finger domain in the $\mathrm{N}$-terminal region and a $\mathrm{C}$-terminal YABBY domain. The genome sequence of the three homologous genes consisted seven exons and six introns. All the introns, except for intron VI, varied in length and sequence composition. Phylogenetic analysis revealed that $T a D L$ was most closely related to the Brachypodium distachyon BdDL and Oryza sativa $D L$ genes. The expression pattern analysis indicated that $T a D L 1, T a D L 2$, and $T a D L 3$ have a higher expression levels in PS and $\mathrm{P}$; however, the three genes are very rare in $\mathrm{S}$. Thus, $T a D L$ gene may contribute to pistil development. Moreover, overexpression of $T a D L$ gene in stamens may cause complete or partial homeosis of stamens into pistils in wheat.
\end{abstract}

Keywords: DROOPING LEAF gene, pistillody line, Droplet Digital PCR, wheat

\section{Introduction}

In the past twenty years, flower development has been the subject of intensive studies, particularly in two dicot plants, Arabidopsis and Antirrhinum (Theissen and Saedler 1999; Theissen 2001). These studies have provided a general understanding on the development of floral organs in higher plants, as explained by the ABC model (Davies and Schwarz-Sommer 1994; Ma 1994; Weigel and Meyerowitz 1994). This model explains how floral organ identity is defined by three classes of homeotic genes, namely, A, B, and C. In this model, each class functions in two adjacent floral whorls: class-A genes specify sepals in the first whorl; A and B genes specify petals in the second whorl; B and C genes specify stamens in the third whorl; and C genes specify carpels in the fourth whorl. Class-C gene plays a central role in specifying carpel identity in flower development, such as the AGAMOUS (AG) gene in Arabidopsis (Yanofsky et al. 1990; Bowman et al. 1991). Loss-of-function mutations in $A G$ result in homeotic transformation of stamens into petals in whorl 3 and carpels into reiterating ag flowers in whorl 4. However, except for the Class-C gene, the YABBY family genes also contribute to carpel development. The DROOPING LEAF (DL) and CRABS CLAW (CRC) genes are members of the YABBY family that encode plant-specific transcription factors with a zinc-finger domain and a helix-loop helix domain (YABBY domain). The $D L$ gene plays two distinct roles in Oryza sativa development: $D L$ is required for formation of the midrib in the leaf and specification of the carpel in the flower (Nagasawa et al. 2003; Yamaguchi et al. 2004; Ohmori et al. 2008). A loss-of function mutation in $D L$ results in a leaf lacking a midrib, the strong structure formed in the central region of the leaf, thereby resulting in drooping leaf phenotypes. In the flower, carpels are homeotically transformed into stamens in the null dl mutant. $C R C$ is an ortholog of the $D L$ gene in Arabidopsis. CRC gene plays a role in carpel identity and nectary development in the flower, and expressed both in the abaxial domains of the carpel primordia and in the nectary (Bowman and Smyth 1999; Baum et al. 2001). MADS box genes, such as B- and C-class floral homeotic genes and SEPALLATA genes, are required for activation of $C R C$ and $D L$ genes (Ohmori et al. 2011). 
The pistillody mutant plant, HTS-1, has been screened from Chinese Spring TP (CSTP), a near-isogenic line of Chinese Spring, which is a common wheat variety carrying the Pis 1 gene derived from the TP mutant (Peng et al 2013). HTS-1 plants exhibit a novel phenotype that transforms all or parts of the stamen into pistils or pistil-like structures. In recent years, the alloplasmic lines of N26 (Murai and Tsunewaki 1993) and (cr)-CSdt7BS (Murai et al. 2002) have been used as research materials to probe the genetic and molecular mechanism of wheat pistillody (Meguro et al. 2003; Mizumoto et al. 2009; Saraike et al. 2007; Zhu et al. 2008). Unclear-cytoplasm interaction (Murai et al. 2002; Zhu et al. 2008) causes the pistillody phenomena of N26 and (cr)-CSdt7BS. However, the pistillody trait of HTS- 1 was determined through interaction of two recessive karyogenes, namely, hts 1 and hts2 (Murai et al. 2002; Zhu et al. 2008). In other words, HTS-1 is genetically different from the previously reported lines of (cr)-CSdt7BS and N26. Wheat floret is considered extremely stable and has few reported mutants. Studies on floret mutants have led in a superficial understanding of the floral organ identity determination in wheat plants. Thus, HTS-1 is a significant material for studying the floral development of wheat, which also has the potential to increase the yield. In a previous study, a sequence from an expressed sequence tag (EST) was initially identified in wheat pistillody line HTS-1 using RNA-seq. Blast searches of this sequence against the GenBank database revealed that it was homologous to the $D L$ gene.

As part of an investigation into the function of the Triticum aestivum DROOPING LEAF gene (TaDL) in pistillody development in this work, we have cloned, characterized, and phylogenetically analyzed the $T a D L$ gene in wheat pistillody line HTS-1. We also examined the expression patterns of this gene in pistillody stamen (PS), the pistil (P) from HTS-1 plants, and the stamen (S) from the non-pistillody control CSTP.

\section{Materials and Methods}

\subsection{Plant Material}

HTS-1 is a common pistillody wheat mutant found in our laboratory. CSTP is a near-isogenic line of Chinese Spring, a common wheat variety that carries the Pis 1 gene derived from the TP mutant (Yang et al. 2012). HTS-1 was selected during the development process of CSTP (Yang et al. 2012). Thus, CSTP and HTS-1 are sib-lines that show similar phenotypes, except for pistillody. CSTP and HTS-1 were cultivated in a field in China West Normal University, Nanchong, China. The PS and P in HTS-1, as well as the S in CSTP at the heading stage, were selected for RNA and DNA extraction.

\subsection{DNA and RNA Isolation}

Genomic DNA was isolated from P of HTS-1 as described by Porebski et al. (1997). The DNA was dissolved in Tris-EDTA (TE) buffer and stored at $-20^{\circ} \mathrm{C}$. Total RNA was isolated from PS, P, and S as described by Manickavelu et al. (2007). The RNA was dissolved in RNase-free double distilled water $\left(\mathrm{ddH}_{2} \mathrm{O}\right)$ and stored at $-70{ }^{\circ} \mathrm{C}$. The quality of the DNA and RNA was confirmed by agarose gel electrophoresis; the nucleic acid concentrations were determined spectrophotometrically based on the $260 / 280 \mathrm{~nm}$ absorbance ratio.

\subsection{Cloning and Chromosomal Mapping of TaDL}

An EST sequence previously identified in the wheat pistillody line HTS-1 using RNA-seq was shown to have functions similar to those of $D L$ gene. Blast search analysis indicated that the full length cDNA (GenBank ID: AB470269) in wheat shared perfect identity with the TaDL EST. The PCR primer pair DL was designed with Primer Premier 5.0 based on the cDNA of AB470269 (Table 1). The cDNA and genomic DNA sequences were amplified using the same primers (DL). PCR amplification was conducted in a thermocycler (My-Cycle, Bio-Rad, San Diego, USA) in a volume of $50 \mathrm{uL}$ containing $100 \mathrm{ng}$ of genomic DNA or reverse transcriptase reaction product (see Droplet Digital PCR part for details), $100 \mu \mathrm{M}$ of each dNTP, $1.5 \mathrm{mM} \mathrm{Mg}^{2+}, 1 \mathrm{U}$ of Taq DNA polymerase, $0.4 \mu \mathrm{M}$ of each primer, and $1 \times \mathrm{PCR}$ buffer. The PCR cycling conditions included pre-denaturation at $94{ }^{\circ} \mathrm{C}$ for $5 \mathrm{~min}$, followed by 35 cycles at $94{ }^{\circ} \mathrm{C}$ for $30 \mathrm{~s}, 60^{\circ} \mathrm{C}$ for $30 \mathrm{~s}$, and $72{ }^{\circ} \mathrm{C}$ for $1 \mathrm{~min}$ or $4 \mathrm{~min}$ (cloning cDNA for $1 \mathrm{~min}$ and DNA for $4 \mathrm{~min}$ ), and a final extension at $72{ }^{\circ} \mathrm{C}$ for $10 \mathrm{~min}$. The amplified products were visualized by gel electrophoresis in $1 \%$ agarose gels, and then documented with a Gel Doc 2000TM system (Bio-Rad). The target DNA bands were recovered and purified from the gels using Qiaquick Gel extraction kits (QIAGEN, Shanghai, China). The purified PCR products were cloned in the pMD-19T vector according to the instructions of the manufacturer (Takata, Dalian, China). Transformants were plated on LB agar containing ampicillin. Clones with inserts were identified using blue/white colony selection. Positive clones were then screened and sequenced by Taihe Biotechnology Co. Ltd. (Beijing, China).

The chromosomal location of TaDL was mapped using the Blast searches of the TaDL genomic sequence against the draft sequence of the bread wheat genome database (https://urgi.versailles.inra.fr/blast/blast.php) (IWGSC, 2014). 


\subsection{Molecular Characterization and Phylogenetic Analysis of TaDL}

The sequence data were analyzed with GenScan software (http://genes.mit.edu/GENSCAN.html). The ORF of the cDNA sequence was searched using the ORF finder software (http://www.ncbi.nlm.nih.gov/gorf/gorf.html). A computation tool of $\mathrm{pl} / \mathrm{Mw}$ for Swiss-Prot/TrEMBL entries (http://www.expasy.org/tools/pi_tool.html) was used to calculate the isoelectric point (PI) and molecular weight (Mw) of TaDL. TaDL was compared with amino acid sequences of other species using Blast 2.1 (www.ncbi.nih.gov/blast/) for homology analysis.

The deduced amino acid sequence of $T a D L$ was aligned with the YABBY family genes reported for other species using the Clustal W program (Thompson et al. 1994). Neighbor-joining trees of the $T a D L$ genes were generated using the MEGA software version 5.0 (Saitou and Nei 1987; Tamura et al. 2007) with 1000 bootstrap replicates. Gaps were treated as missing values. The amino acid sequence of the YABBY family genes from Arabidopsis thaliana (CRC: Q8L925, YAB3: Q9XFB1), Oryza sativa (DL: AAR84663, OsYAB3: Q0JBF0 and OsFIL: AF098752), Zea mays (ZmDL1: NP_001148730), Triticum aestivum (TaYAB1: AAQ93323), Brachypodium distachyon (BdDL: XP_010228969), Solanum lycopersicum (SlCRC: XP_004239032), and Nicotiana sylvestris (YABBY 5: XP_009762053) were obtained from NCBI.

\subsection{Droplet Digital PCR (ddPCR)}

Total RNA was isolated from PS, P, and S. The cDNA was synthesized using the iScript Select cDNA synthesis kit (Bio-Rad, Hercules, USA). The primers and probes for TaDL1, TaDL2, and TaDL3 were designed using Beacon Designer (Table1 and Figure 1). The primer pair d1 and probe p1 can amplify TaDL1, TaDL2, and $T a D L 3$. However, the primer pair $\mathrm{d} 2$ and probe $\mathrm{p} 2$ can only amplify $T a D L 2$, while primer pair $\mathrm{d} 3$ and probe $\mathrm{p} 3$ can only amplify $T a D L 3$. The wheat housekeeping gene beta-actin (AB181991) plays the role of internal control (Yamada et al. 2009). For ddPCR, the reaction mixture was assembled from a $2 \times$ ddPCR Supermix (Bio-Rad), plus primers ( $250 \mathrm{nM}$ each), probes $(125 \mathrm{nM})$, and $1 \mu \mathrm{L}$ cDNA in a final volume of $20 \mu \mathrm{L}$ for $T a D L$ and internal control gene. Droplet generation, PCR reaction, and detection were carried out according to instructions of the manufacturer. Briefly, each assembled ddPCR reaction mixture was loaded into the sample well of an eight-channel droplet generator cartridge (Bio-Rad). A $70 \mu \mathrm{L}$ volume of droplet generation oil was then loaded into the oil well for each channel. The cartridge was placed into the droplet generator (Bio-Rad) for droplet generation (up to 20,000 per reaction). The droplets collected in the droplet well of the cartridge were then manually transferred with a multichannel pipette to a 96-well PCR plate. The plate was heat-sealed with a foil seal, and then placed on a conventional thermal cycler (T100, Bio-Rad). After PCR, the 96-well PCR plate was loaded on the droplet reader (QX200, Bio-Rad), which automatically reads the droplets from each well of the plate. Using auto-analysis after data acquisition with QuantaSoft analysis software. The expression quantitation of $T a D L 2$ and $T a D L 3$ can be obtained directly using the following formula: $T a D L 1=$ the total expression of $T a D L 1, T a D L 2$, and $T a D L 3$ (expression quantitation use pair $\mathrm{d} 1$ and probe $\mathrm{p} 1)-(T a D L 2+T a D L 3)$. The absolute expression quantitation of these genes was normalized using internal control gene.

\section{Results}

\subsection{Cloning cDNA and Genomic Sequences}

The cDNA from P of HTS-1 plants amplified with the DL primer pair yielded a fragment of $\sim 900 \mathrm{bp}$. The PCR product was cloned into the PMD-19T vector; 15 clones were sequenced. Three distinct sequences of 879, 885, and $876 \mathrm{bp}$ were identified, indicating that the band of $\sim 900 \mathrm{bp}$ included comigrating cDNA of different homologous genes. The three homologous genes shared $97.79 \%$ homology with each other. We tentatively designated the three homologous genes as TaDL1, TaDL2, and TaDL3. The TaDL1, TaDL2, and TaDL3 share the same length of 3'-untranslated region (3'-UTR), namely, $147 \mathrm{bp}$. The 5'-untranslated region (5'-UTR) of TaDL1, $T a D L 2$, and $T a D L 3$ were 129, 129, and 126 bp, respectively. The ORF of TaDL1, TaDL2, and TaDL3 were 603, 609 , and 603bp, coded for deduced protein of 200, 202 and 200 amino acids, respectively. Primary structure analysis using Swiss-Prot/TrEMBL revealed that the molecular mass of the putative TaDL1, TaDL2, and TaDL3 protein were $22.4,22.6$, and $22.3 \mathrm{kDa}$, with theoretical $\mathrm{pI}$ of 9.04, 9.04, and 8.98, respectively. BLAST searches showed that $T a D L 1, T a D L 2$, and TaDL3 belonged to the YABBY family. Sequence alignment suggested that $T a D L$ had high homology with other plant DL proteins, which contain two distinct domains: a zinc-finger domain in the N-terminal region and a C-terminal YABBY domain (helix-loop-helix domain) (Figure 2).

Approximately 4000 bp DNA fragment was amplified with primers DL-F and DL-R. Cloning and analyzing the PCR products, three different genomic sequences, 4284, 4050, and 4128bp long, were isolated from P of HTS-1 plants. The three genomic sequences shared $88.8 \%$ homology with each other. 


\subsection{Structure and Chromosomal Location of TaDL Genomic Sequence}

The TaDL1, TaDL2, and TaDL3 genomic sequences were 4284, 4050, and 4128 bp long, respectively. Their alignment and comparison with the corresponding cDNA sequences revealed the complex structure of the three homologous genes consisting seven exons and six introns (Figure 3). The six introns fulfilled the GT-AG rule, but varied markedly in length and sequence composition. The length of intron IV was 1688, 1476, and 1565bp in $T a D L 1, T a D L 2$, and $T a D L 3$, respectively, which was longer than the other five introns. Intron VI was 98bp long in the three genes and was the shortest among the six introns. Intron I was 585, 586, and 583bp, respectively. Intron II had three lengths, namely, 724, 708, and 699bp. Introns III and V showed two lengths (intron III was 191 and 189 bp; intron V was 119 and 118bp) in TaDL1, TaDL2, and TaDL3.

Blast searches of the $T a D L$ genomic sequence against the draft sequence of the bread wheat genome database (https://urgi.versailles.inra.fr/blast/blast.php) (IWGSC, 2014) revealed that TaDL1, TaDL2, and TaDL3 were 100\% homologous to the IWGSC_chr4AS_V2_ab_k71_contigs_longerthan_200_6011626, IWGSC_chr4DL_V3_ab_k71_contigs_longerthan_200_14449099, and IWGSC_chr4BL_ab_k71_contigs_longerthan_200_5583565, respectively. Therefore, TaDL1 may be located on the short arm of chromosome $4 \mathrm{~A}$, whereas $T a D L 2$ and $T a D L 3$ are located on the long arm of chromosome 4D and $4 \mathrm{~B}$, respectively.

\subsection{Phylogenetic Analysis of TaDL and plant YABBY Family Gene}

YABBY from A. thaliana (CRC and YAB3), O. sativa (DL, OsYAB3 and OSFIL), Z. mays (ZmDL1), T. aestivum (TaYAB1), B. distachyon (BdDL), S. lycopersicum (SlCRC), and N. sylvestris (YABBY 5) were analyzed to construct the phylogenetic tree. Figure 4 shows the tree obtained through the neighbor joining program using MEGA software and 1000 bootstrap replicates. Previous analysis identified three types of YABBY in plants, namely, FIL subfamily, DL/CRC subfamily, and INO subfamily (Yamaguchi et al., 2004). The INO subfamily is a small family in plants, including only the $I N O$ gene. In the present study, these YABBY genes formed into two well-resolved subfamily: the FIL subfamily, including A. thaliana YAB3 gene, O. sativa OsYAB3 and OsFIL gene, T. aestivum TaYAB1 gene, and N. sylvestris YABBY 5 gene; and the DL/CRC subfamily, including T. aestivum $T a D L 1, T a D L 2$, and TaDL3 gene, B. distachyon BdDL gene, O. sativa $D L$ gene, Z. mays ZmDL1 gene $S$. lycopersicum SlCRC, and A. thaliana CRC gene (Figure 4). The three $T a D L$ genes in this study were located in the DL/CRC subfamily and showed much greater similarity (more than $85 \%$ ) to the $B$. distachyon $B d D L$ and $O$. sativa $D L$ genes.

\subsection{TaDL Gene Expression Patterns in P, PS and S}

ddPCR was used to study the pattern of TaDL1, TaDL2, and TaDL3 expression in PS, P, and S. As shown in Figure 5, TaDL1, TaDL2, and TaDL3 have a higher expression levels in PS and P, but very rare in S. The TaDL1 gene in $\mathrm{P}$ is approximately 2-fold higher than PS; however, TaDL2 and TaDL3 have the highest expression level in PS (about 4 and 2.3 fold higher than P, respectively). In the three genes, TaDL3 has the highest expression level in P (about 138061 copies) and PS (about 317714 copies).

\section{Discussion}

An EST sequence previously identified in the wheat pistillody line HTS-1 using RNA-seq was shown to have functions similar to those of $D L$ gene. The EST was tentatively designated as a $T a D L$ gene. In this study, cDNA cloning and genome sequence of $T a D L$ from wheat showed that three homologous genes, namely, $T a D L 1$, $T a D L 2$, and $T a D L 3$. BLAST searches showed that the $T a D L$ gene belonged to the YABBY gene family and contained two distinct domains: a zinc-finger domain in the N-terminal region and a C-terminal YABBY domain.

YABBY gene family was classified into three subfamilies, namely, FIL subfamily, $D L / C R C$ subfamily, and INO subfamily (Yamaguchi et al. 2004). The TaDL genes in this study belong to the DL/CRC subfamily. $C R C$ is an ortholog of $D L$ in Arabidopsis, plays a role in carpel identity and nectary development in the flower, and expressed both in the abaxial domains of the carpel primordia and in the nectary (Bowman and Smyth 1999; Baum et al. 2001). Unlike rice $D L, C R C$ appears to have no function in vegetative development in Arabidopsis. Thus, the regulation of carpel development seems to be an ancestral function of $D L$ and $C R C$ (Ohmori et al. 2011). $C R C$ has binding sites for LFY and MADS domain proteins in its 5 ' upstream region; the latter site may be essential for the transcriptional regulation of $C R C$ (Lee et al. 2005). MADS box genes such as B- and C-class floral homeotic genes and SEPALLATA genes are required for $C R C$ activation. Alignment and phylogenetic analysis demonstrated that $T a D L$ shared more identity with the $B$. distachyon $B d D L$ and $O$. sativa DL genes. These finding suggest that $T a D L$ may have similar biological roles to $B d D L$ and $D L$ genes. The function of 
$B d D L$ remains unclear; however, a severe or intermediate mutation in $D L$ in rice causes complete or partial homeotic of carpels into stamens without affecting the identities of the other floral organs (Yamaguchi et al. 2004).

Common wheat is an allohexaploid composed of three distinct ancestral genomes, namely, A, B and D. In this study, three distinct $T a D L$ homologous genes were be identified. Chromosomal location analysis indicated that the TaDL1 is located on $4 \mathrm{AS}$, while TaDL2 and TaDL3 are located on $4 \mathrm{DL}$ and $4 \mathrm{BL}$, respectively. The three homologous genes come from different ancestral genomes, so the DNA and cDNA sequence showed certain degree of differences: the cDNA of TaDL3 deleted 4bp in the site of 67bp, and TaDL1 and TaDL3 deleted 6bp in the site of 203bp (Figure 1). Accurately determining the expression of TaDL1, TaDL2, and TaDL3 in PS, P, and $\mathrm{S}$ using real-time PCR is difficult because the real-time PCR detected relative expression quantity of those genes. The relative expression quantitation cannot be added or subtracted to each other. However, ddPCR enables the absolute quantitation of nucleic acids in a sample; thus, expression quantitation can be added or subtracted to each other. For example, the primer pair d1 and probe p1 can amplify TaDL1, TaDL2, and TaDL3, whereas the primer pair $\mathrm{d} 2$ and probe $\mathrm{p} 2$ can only amplify $T a D L 2$ and primer pair $\mathrm{d} 3$ and probe $\mathrm{p} 3$ can only amplify $T a D L 3$. Thus, the expression quantitation of $T a D L 2$ and $T a D L 3$ can be obtained directly. Calculating the expression quantitation of $T a D L 1$ is difficult using real-time PCR; however, can be easily conducted using ddPCR and the following formula: $\mathrm{TaDL} 1=$ the total expression of TaDL1, TaDL2, and TaDL3- $(T a D L 2+T a D L 3)$. The absolute expression quantitation of those genes were normalized using internal control gene to obtain the relative expression quantitation of $T a D L 1, T a D L 2$, and $T a D L 3$ in PS, P, and S. Meanwhile, ddPCR allows for precise quantification of nucleic acids, facilitating the measurement of small percentage differences and quantification of rare variants. ddPCR may also be more reproducible and less susceptible to inhibition than real-time PCR (Sun et al. 2014). In this study, the expression of $T a D L 1, T a D L 2$, and $T a D L 3$ in PS, P, and $\mathrm{S}$ was analyzed use ddPCR. The results indicated that $T a D L 1, T a D L 2$, and $T a D L 3$ have a higher expression levels in PS and P, but very rare in S. Based on these observations, we suggest that $T a D L 1, T a D L 2$, and $T a D L 3$ may have the same functions and importantance in defining pistil development. Overexpression of the $T a D L$ gene in stamens may cause complete or partial homeosis of stamens into pistils in wheat. However, this suggestion requires confirmation through additional experiments.

Table 1. Primers and probes used in this study

\begin{tabular}{lllll}
\hline PCR target & Primer & Forward & Reverse & probes \\
\hline Genomic and & DL & CAGCAACGAAGTAACCAAACAT & CCTCCTACCCTCCACATCCTC & \\
cDNA sequence & & & & FAM-TCGCCGTCCGAGCACCTCTG-BHQ1 \\
ddPCR & $\mathrm{d} 1$ & GTGTCCATCAGCCTCTTG & CTCACTCTTGACCTCTCC & FAM-ACCCCAACCTGCAGCGCGAG-BHQ1 \\
& $\mathrm{d} 2$ & GTGTCCATCAGCCTCTTG & CTCACTCTTGACCTCTCC & FAM-TCTTGACTTCTCCCACCGACG-BHQ1 \\
& $\mathrm{d} 3$ & CACCAAATCCATGCTCTG & CGTCTTCTATTGGCTCTTC & FAM-TGGAGAAGAGCTATGAGATGC-BHQ1 \\
\hline
\end{tabular}

$\mathrm{D} 1 / \mathrm{D} 2-\mathrm{F}$

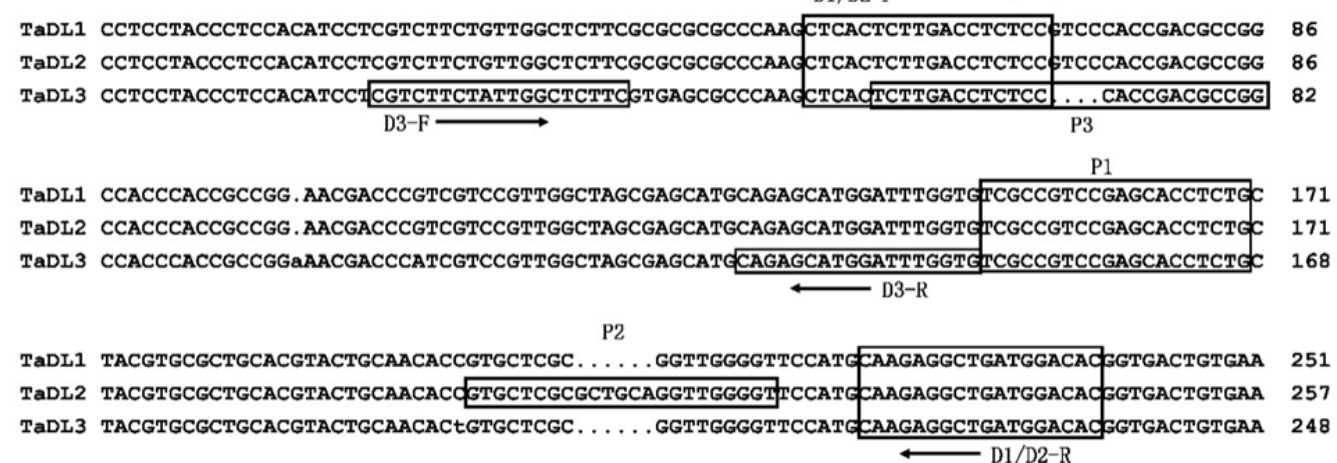

Figure 1. Alignment of the partial cDNA sequences TaDL1, TaDL2 and TaDL3. The primers and probes used for ddPCR are boxed 


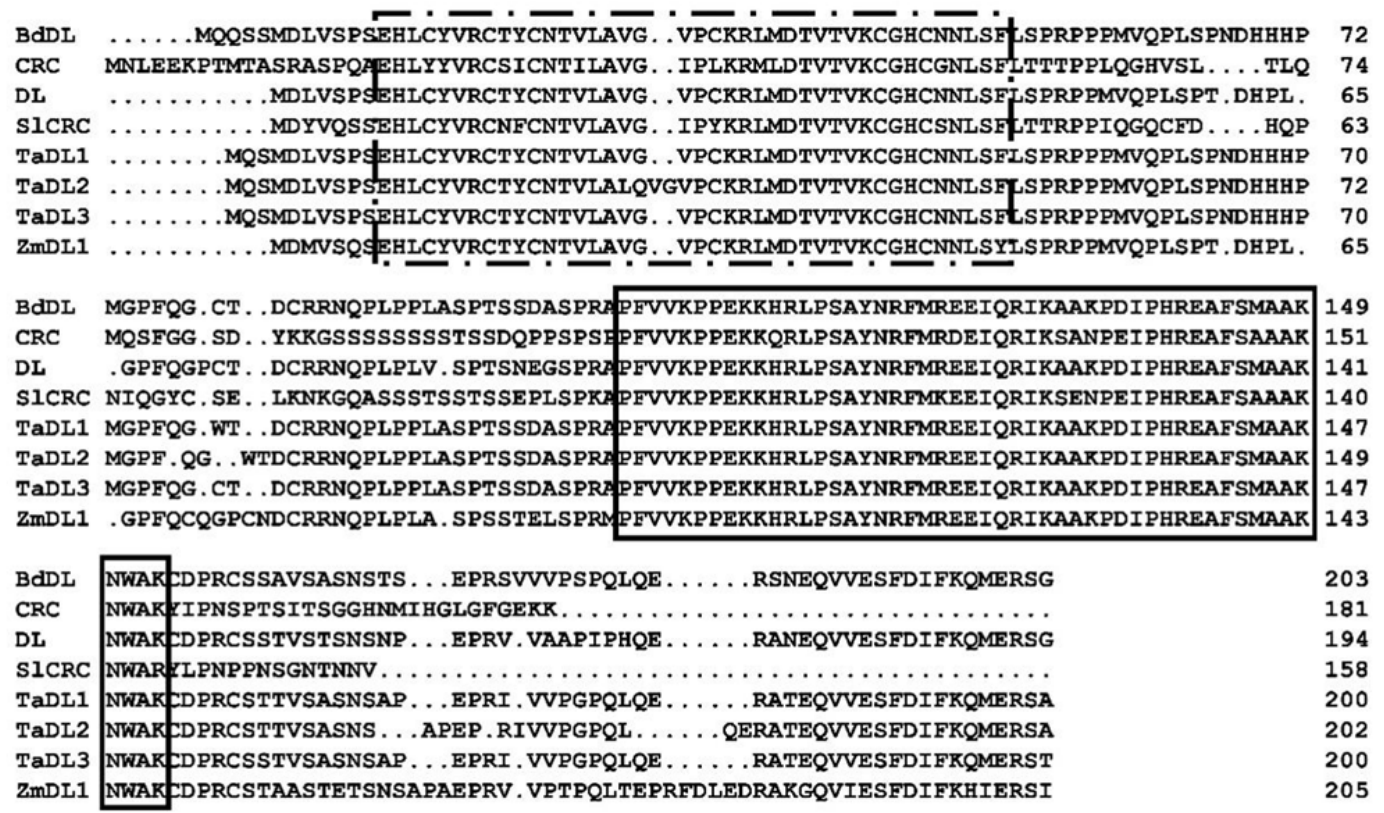

Figure 2. Aligment of the deduced amino acid sequence of the TaDL with other plant DL sequences. Zinc-finger domain and C-terminal YABBY domain are indicated by the dotted line and solid line frames, respectively

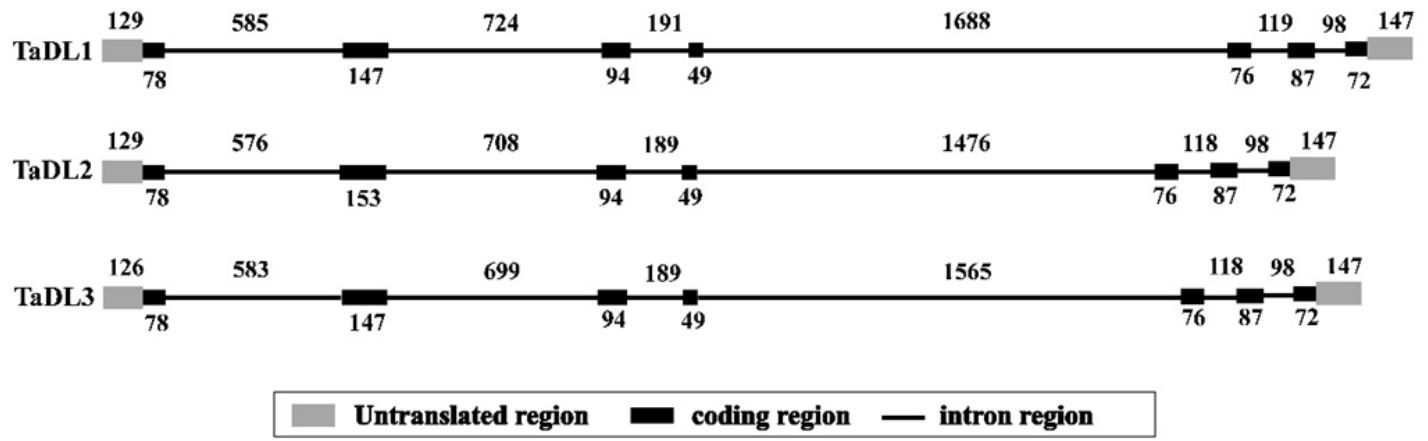

Figure 3. Schematic representation of the TaDL gene. 


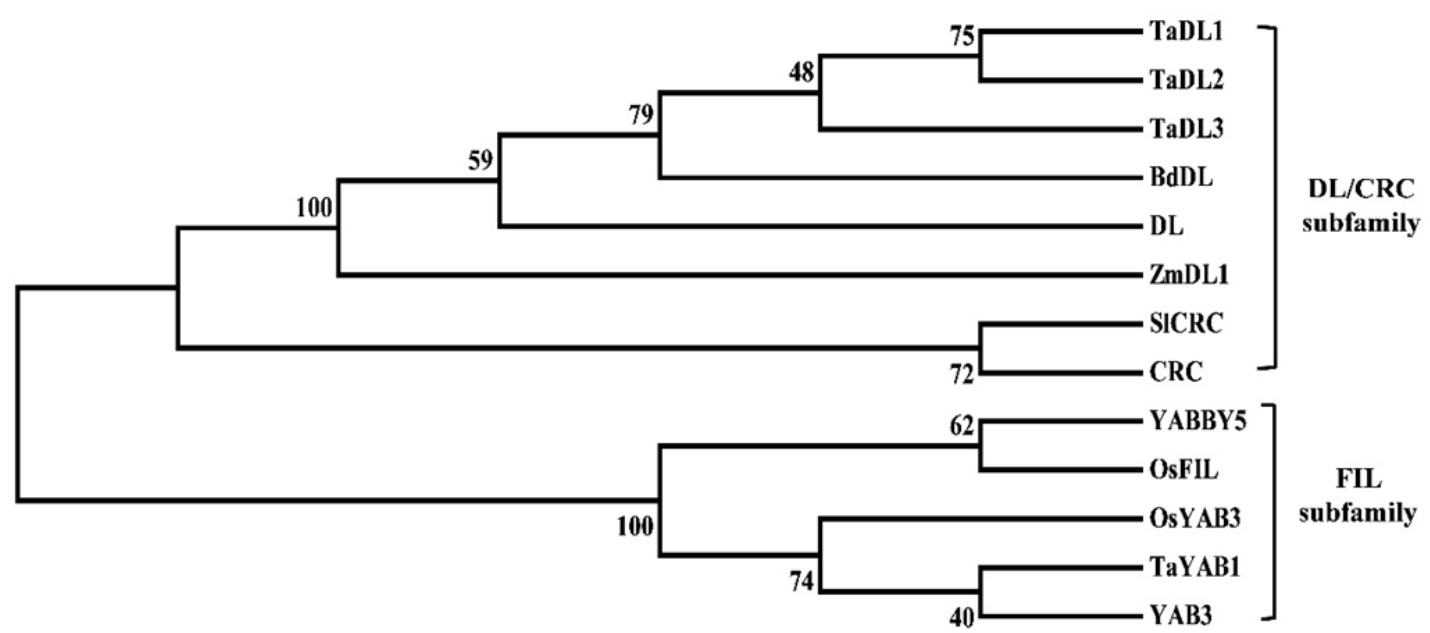

Figure 4. Phylogenetic tree of TaDL and other homologous sequences. The numbers at the nodes indicate bootstrap values

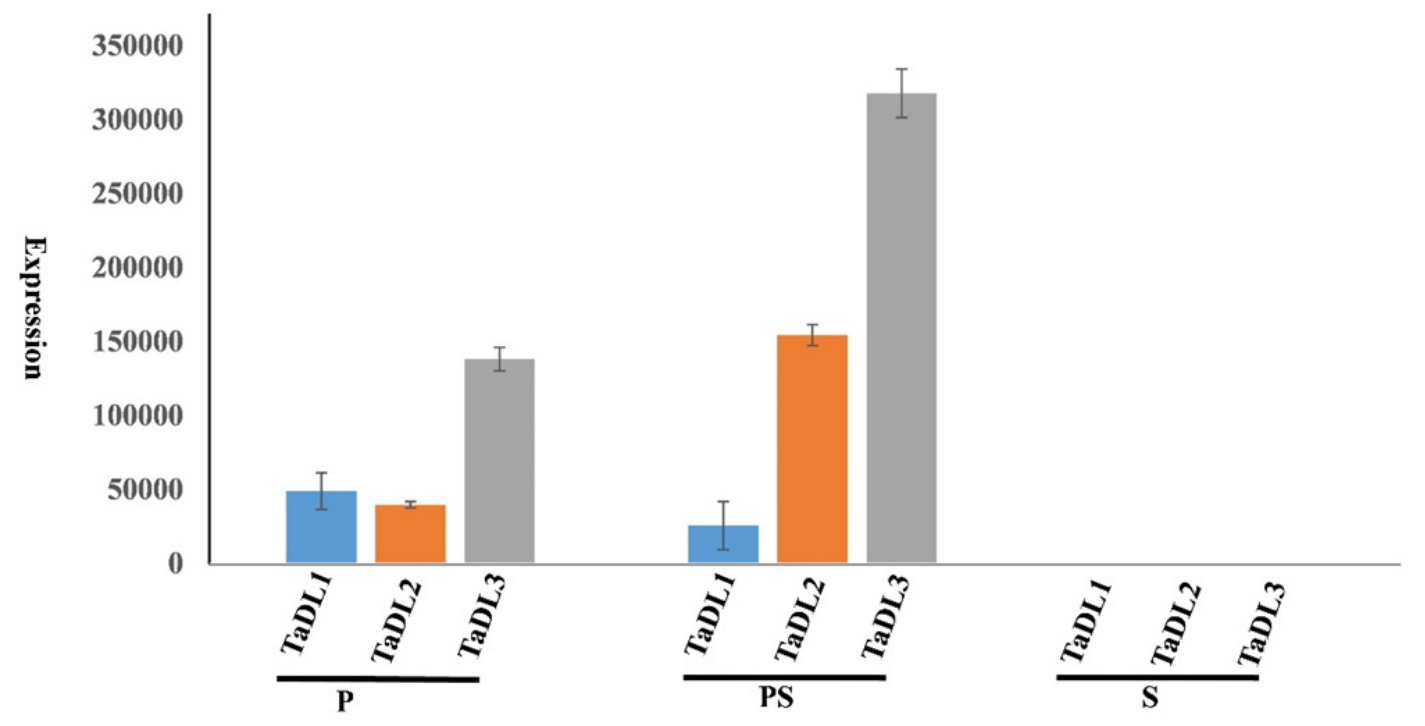

Figure 5. TaDL expression assessed by ddPCR. $\beta$-actin were used as internal controls. The transcript levels are shown as relative values

\section{Acknowledgments}

This work was financially supported by the National Natural Science Foundation of China (Grant No. 31301319), the Key Project of the Chinese Ministry of Education (Grant No. 211164), and the Scientific Research Fund of Sichuan Provincial Education Department (Grant No. 12ZB325). We thank Yun Zhao and Yanfei Chen, as well as all the staff of Bio-Rad Laboratories, Inc., for their help in Droplet Digital PCR.

\section{References}

Baum, S. F., Eshed, Y., \& Bowman, J. L. (2001). The Arabidopsis nectary is an ABC-independent floral structure. Development, 128, 4657-4667.

Bowman, J. L., \& Smyth, D. R. (1999). CRABS CLAW, a gene that regulates carpel and nectary development in Arabidopsis, encodes a novel protein with zinc finger and helix-loop-helix domains. Development, 126, 
2387-2396.

Bowman, J. L., Drews, G. N., \& Meyerowitz, E. M. (1991). Expression of the Arabidopsis floral homeotic gene AGAMOUS is restricted to specific cell types late in flower development. Plant Cell, 3, 749-758.

Davies, B., \& Schwarz-Sommer, Z. (1994). Control of floral organ identity by homeotic MADS-box transcription factors. In Nover L (Ed.), Plant promoters and transcription factors (pp. 235-258). Springer, Berlin Heidelberg New York.

Lee, J. Y., Baum, S. F., Alvarez, J., Patel, A., Chitwood, D. H., \& Bowman, J. L. (2005). Activation of CRABS CLAW in the nectaries and carpels of Arabidopsis. Plant Cell, 17, 25-36. http://dx.doi.org/ $10.1105 /$ tpc. 104.026666

Ma, H. (1994). The unfolding drama of flower development: recent results from genetic and molecular analyses. Genes \& development, 8, 745-756.

Manickavelu, A., Kambara, K., Mishina, K., \& Koba, T. (2007). An efficient method for purifying high quality RNA from wheat pistils. Colloids Surf $B$ Biointerfaces, 54, 254-258. http://dx.doi.org/10.1016/j.colsurfb.2006.10.024

Meguro, A., Takumi, S., Ogihara, Y., \& Murai, K. (2003). WAG a wheat AGAMOUS homolog, is associated with development of pistil-like stamens in alloplasmic wheats. sexual plant reproduction, 15, 221-230.

Mizumoto, K., Hatano, H., Hirabayashi, C., Murai, K., \& Takumi, S. (2009). Altered expression of wheat AINTEGUMENTA homolog, WANT-1, in pistil and pistil-like transformed stamen of an alloplasmic line with Aegilops crassa cytoplasm. Development genes and evolution, 219, $175-187$. http://dx.doi.org/10.1007/s00427-009-0275-y

Murai, K., \& Tsunewaki, K. (1993). Photoperiod-sensitive cytoplasmic male sterility in wheat with Aegilops crassa cytoplasm. Euphytica, 67, 41-48. http://dx.doi.org/10.1007/BF00022723

Murai, K., Takumi, S., Koga, H., \& Ogihara, Y. (2002). Pistillody, homeotic transformation of stamens into pistil-like structures, caused by nuclear-cytoplasm interaction in wheat. The Plant Journal, 29, 169-182.

Nagasawa, N., Miyoshi, M., Sano, Y., Satoh, H., Hirano, H., Sakai, H., \& Nagato, Y. (2003). SUPERWOMAN1 and DROOPING LEAF genes control floral organ identity in rice. Development, 130, 705-718. http://dx.doi.org/10.1242/dev.00294

Ohmori, Y., Abiko, M., Horibata, A., \& Hirano, H. Y. (2008). A transposon, Ping, is integrated into intron 4 of the DROOPING LEAF gene of rice, weakly reducing its expression, and causing a mild drooping leaf phenotype. Plant and Cell Physiology, 49, 1176-1184. http://dx.doi.org/10.1093/pcp/pcn093

Ohmori, Y., Toriba, T., Nakamura, H., Ichikawa, H., \& Hirano, H. Y. (2011). Temporal and spatial regulation of DROOPING LEAF gene expression that promotes midrib formation in rice. The Plant Journal, 65, 77-86. http://dx.doi.org/10.1111/j.1365-313X.2010.04404.x

Peng, Z. S., Yang, Z. J., Ouyang, Z. M., \& Yang, H. (2013). Characterization of a novel pistillody mutant in common wheat. Australian Journal of Crop Science, 7, 159-164.

Porebski, S., Bailey, L. G., \& Baum, B. R. (1997). Modification of a CTAB DNA extraction protocol for plants containing high polysaccharide and polyphenol components. Plant Molecular Biology Reporter, 15, 8-15. http://dx.doi.org/10.1007/BF02772108

Saraike, T., Shitsukawa, N., Yamamoto, Y., Hagita, H., Iwasaki, Y., Takumi, S., \& Murai, K. (2007). Identification of a protein kinase gene associated with pistillody, homeotic transformation of stamens into pistil-like structures, in alloplasmic wheat. Planta, 227, 221. http://dx.doi.org/10.1007/s00425-007-0608-x

Sun, B., Tao, L., \& Zheng, Y. L. (2014). Simultaneous quantification of alternatively spliced transcripts in a single droplet digital PCR reaction. BioTechniques, 56, 319-325.

Theissen, G., \& Saedler, H. (1999). The golden decade of molecular -oral development (1990-99): a cheerful obituary. Genes \& Development, 25, 1-13.

Theissen, G. (2001). Development of floral organ identity: stories from the MADS house. Current Opinion in Plant Biology, 4, 75-85.

Thompson, J. D., Higgins, D. G., \& Gibson, T. J. (1994). CLUSTAL W: improving the sensitivity of progressive multiple sequence alignment through sequence weighting, position-specific gap penalties and weight matrix 
choice. Nucleic Acids Research, 22, 4673-4680. http://dx.doi.org/10.1093/nar/22.22.4673

Weigel, D., \& Meyerowitz, E. M. (1994). The ABCs of floral homeotic genes. Cell, 78, 203-209. http://dx.doi.org/10.1016/0092-8674(94)90291-7

Yamada, K., Saraike, T., Shitsukawa, N., Hirabayashi, C., Takumi, S., \& Murai, K. (2009). Class D and B MADS-box genes are associated with ectopic ovule formation in the pistil-like stamens of alloplasmic wheat (Triticum aestivum L.). Plant Molecular Biology Reporter, 71, 1-14. http://dx.doi.org/10.1007/s11103-009-9504-z

Yamaguchi, T., Nagasawa, N., Kawasaki, S., Matsuoka, M., Nagato, Y., \& Hirano, H. Y. (2004). The YABBY gene DROOPING LEAF regulates carpel specification and midrib development in Oryza sativa. Plant Cell, 16, 500-509. http://dx.doi.org/10.1105/tpc.018044

Yang, Z. J., Peng, Z. S., Zhou, Y. H., Peng, L. J., \& Wei, S. H. (2012). Evaluation on the genetic background of near isogentic lines for three pistils character by SRAP marks. Journal of Nuclear Agricultural Sciences, 26 , 22-27.

Yanofsky, M. F., Ma, H., Bowman, J. L., Drews, G. N., Feldmann, K. A., \& Meyerowitz, E. M. (1990). The protein encoded by the Arabidopsis homeotic gene agamous resembles transcription factors. Nature, 346, 35-39. http://dx.doi.org/10.1038/346035a0

Zhu, Y., Saraike, T., Yamamoto, Y., Hagita, H., Hagita, H., Takumi, S., \& Murai, K. (2008). Orf260cra, a Novel mitochondrial gene, is associated with the homeotic transformation of stamens into pistil-like structures (Pistillody) in alloplasmic wheat. Plant and Cell Physiology, 49, 1723-1733.

\section{Copyrights}

Copyright for this article is retained by the author(s), with first publication rights granted to the journal.

This is an open-access article distributed under the terms and conditions of the Creative Commons Attribution license (http://creativecommons.org/licenses/by/3.0/). 